\title{
Fetal Growth Restriction and Neurodevelopmental Outcome
}

\author{
Deepak Chawla ${ }^{1}$ (D)
}

Received: 22 April 2021 / Accepted: 26 April 2021 / Published online: 30 April 2021

(C) Dr. K C Chaudhuri Foundation 2021

Fetal growth restriction (FGR) is an important public health problem in India [1]. High incidence of FGR in India is related to a younger age at pregnancy, reduced interpregnancy interval, maternal low birth weight, and maternal macro- and micronutrient deficiency. FGR is a significant risk factor of stillbirth, birth asphyxia, meconium aspiration syndrome, hypoglycemia, hypothermia, and systemic sepsis. Preterm neonates born with FGR are also at a greater risk of developing hyaline membrane disease, bronchopulmonary dysplasia, necrotizing enterocolitis, and retinopathy of prematurity. Compared to their appropriate-for-gestation age (AGA) counterparts, preterm small-for-gestation (SGA) neonates are also at greater risk of long-term neurological complications, including cerebral palsy, delayed development, behavioral disorders, and learning disabilities. Magnetic resonance imaging (MRI) studies have reported lower total and regional brain volume in neonates with FGR [2]. Various areas of brain reported to be affected in different studies include thalamus, cerebrum, putamen, brainstem, hippocampus, and cerebellum. In a systematic review including 60 studies and 52,822 neonates, Sacchi et al. have reported lower cognitive scores in both term and preterm neonates with FGR [3]. In another systematic review, Levine et al. included 16 studies [4]. Of these, 11 studies reported worse neurological outcome in FGR. However, the domain affected varied in different studies - motor delay was reported in 10 studies, cognitive delay was reported in 8 studies, and language delay was reported in 7 studies.

Kohat et al. in their study published in this issue of the Journal, compared neurodevelopmental outcome of 70 preterm FGR neonates with gestation- and gender-matched control neonates [5]. The primary outcome of death or neurodevelopmental disability at $1 \mathrm{y}$ of age was almost three times (RR: 2.83; 95\% CI: 1.11-7.18) more common in FGR

Deepak Chawla

drdeepakchawla@gmail.com

1 Department of Neonatology, Government Medical College Hospital, Chandigarh 160 030, India neonates. While FGR neonates had lower motor development index score at $1 \mathrm{y}$ of age, the mental development index was similar to control infants. The accumulative evidence suggests two things. First, the neonates with FGR are more likely to have adverse neurodevelopmental outcome and simple labeling of neonates as preterm or term SGA and symmetrical or asymmetrical growth restriction may not be sufficient to identify neonates at risk of the adverse outcome. Although, MRI of brain at term-equivalent age may be able to identify neonates with lower global or regional brain volumes, the sheer number of FGR neonates makes it practically impossible to subject every candidate to neuroimaging. More research is needed to identify risk factors of adverse neurological outcome including markers of severity and duration of fetal hypoxia-ischemia. Second, variability both in the region of brain involved and the functional domain affected in different studies indicates role of effect modifiers or confounding factors. Many of the neonatal morbidities mentioned above are observed more commonly in FGR than AGA neonates and are independently associated with adverse neurodevelopmental outcome. In the study by Kohat et al., 4 cases developed severe neurodevelopmental disability and all 4 had alternate sufficient risk factors of severe neurodevelopmental disability. Authors were unable to conduct an adjusted analysis due to low event rate and small sample size. Larger studies are needed to evaluate incidence, risk factors, and types of adverse neurodevelopmental outcome in neonates with FGR. Such a cohort when established should be followed up till adulthood to evaluate long-term effects.

\section{Declarations}

Conflict of Interest None.

\section{References}

1. Lee AC, Katz J, Blencowe H, et al. National and regional estimates of term and preterm babies born small for gestational age in 138 low- 
income and middle-income countries in 2010. Lancet Glob Health. 2013;1:e26-36.

2. Bruno CJ, Bengani S, Gomes WA, et al. MRI differences associated with intrauterine growth restriction in preterm infants. Neonatology. 2017;111:317-23.

3. Sacchi C, Marino C, Nosarti C, Vieno A, Visentin S, Simonelli A. Association of intrauterine growth restriction and small for gestational age status with childhood cognitive outcomes: a systematic review and meta-analysis. JAMA Pediatr. 2020;174:772-81.

4. Levine TA, Grunau RE, McAuliffe FM, Pinnamaneni R, Foran A, Alderdice FA. Early childhood neurodevelopment after intrauterine growth restriction: a systematic review. Pediatrics. 2015;135:12641.

5. Kohat D, Keepanasseril A, Chinnakali P, Mondal N, Bhat BV. Neurodevelopmental outcome of preterm newborns with abnormal umbilical artery doppler - a prospective cohort study. Indian J Pediatr. 2020. https://doi.org/10.1007/s12098-020-03547-x.

Publisher's Note Springer Nature remains neutral with regard to jurisdictional claims in published maps and institutional affiliations. 